\title{
Interventional calendar
} and protocol for cleft lift and palate repair at the maxillofacial and plastic surgery department of the

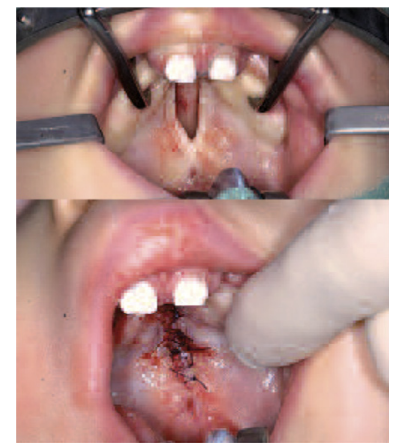

\section{Armand Trousseau Children's Hospital (AP-HP, Paris)}

Arnaud PICARD, Eva GALLIANI, Véronique SOUPRE, Natacha KADLUB, Sophie CASSIER, Georgiana CONSTANTINESCU, Frédéric ZAZURCA, Catherine TOMAT, Brigitte VI-FANE, Chantal TRICHET-ZBINDEN, Cécile CHAPUIS-VANDENBOGAERDE, Patrick A. DINER, Marie-Paule VAZQUEZ

\section{ABSTRACT}

Parents want their children's' faces, the most visible part of their bodies, one that marks their identity throughout life, to be perfect. To satisfy this understandably urgent desire, a high quality of primary treatment for cleft lip and palate is essential and must satisfy a double objective: restore normal morphology and normal function. The functional, morphological, and esthetic prognoses depend on the character of the defect, whether it stands alone or is associated in a syndrome with other malformations. Important sequellae flow from the quality of the initial repair, as a consequence of the surgery and other therapies as well as from the deformity itself.

Before the year 2000, the Maxillo-facial and Plastic Surgery Service at the Armand Trousseau Hospital of the Pierre and Marie Curie Faculty of Medicine 
adhered to the protocol that Malek had described, making an initial repair of the soft palate at 3 months and then a cheiloplasty, with upper and lower triangles, and closure of the hard palate at 6 months. Since then we have adopted the more functional approach that Talmant described, integrating systematic nasal surgery and the type of lip surgery that Millard suggested without leaving any residual exposed bone after closure of the osseous cleft. We then perform gingivo-periosteal surgery with bone grafts on patients when they were between 4 and 6 years of age, after orthodontic therapy had been completed. This constitutes the last stage of primary treatment.

The therapeutic approach we have been using on our service, which has evolved of over the last 20 years, has come to define its principal objective as integration of extensive rehabilitation into the very first stages of our multi-disciplinary therapy so as to minimize the establishment of faulty functioning of phonation, lip competence, and ventilation while avoiding any intervention that would have a harmful impact on facial growth. This multi-disciplinary approach, which integrates surgical evaluation and protocol, is indispensable and fully justifies treatment of patients with cleft lip and palate at accredited centers.

\section{KEYWORDS}

Cleft lip,

Cleft palate,

Bone graft,

Orthodontics,

Rhinoplasty.

\section{1 - INTRODUCTION}

Although all inter-disciplinary teams treating labio-palatal clefts share the same objectives, there is at present no national or international consensus on what procedures to employ in seeking those goals nor on what chronology should be used. In 2000, a Europe-wide inquiry on the subject confirmed this lack of agreement when it received 194 different protocols from 201 centers $^{4}$.
The face, the most visible feature of a human's body, one that doting parents dream will be perfect, marks a person's identity throughout life. Medical management of facial deformities, like clefts, begins prenatally when ultrasound examinations discern them. In these cases, the surgeon's first task is to break the disquieting news to expectant parents and help them prepare for the arrival 
of their very special child. Adequate preparation for these future parents on the fundamentals of the treatment their newborn will have to receive will, without any doubt, reduce the anguish they will have to endure and, also, will ameliorate the psychological distress the child itself will ultimately be subjected to.

It is essential that treating teams offer a high quality of initial treatment

\section{2 - TREATMENT PROTOCOL}

Even though we continue to emphasize our objective of minimizing sequellae by diligently restoring the functions of phonation, mastication, and ventilation, and avoiding any procedure that could have a deleterious effect on growth, we have modified our therapeutic approach over the past 10 years. We have adopted the functional procedures that Talmant ${ }^{6,7}$ described.

\section{2 - 1 - Prenatal management}

The diagnosis of a labio-palatal cleft is most often made in a prenatal morphological ultrasound examination during the second trimester of pregnancy. We then discuss it at our Multidisciplinary Prenatal Diagnostic Center, evaluating any available supplementary radiological and genetic records searching for possible associated anomalies. In a consultation with the surgeon our group confirms the diagnosis and establishes a prognosis for the deformity. Then, in a prenatal appointment, using ultrasound documents, we explain the various for patients with labio-palatal clefts. By providing patients with reconstruction of cutaneo-mucosal, muscular, and cartilaginous structures surgeons will restore functional activity, proper morphology, and acceptable esthetics for them. The quality of this rehabilitation will also greatly affect the nature of later sequellae that are most often caused by some surgical lapse rather than by the original malformation.

dimensions of the deformity to the prospective parents describing what the surgical and later over-all management of the newborn will be. Next, parents have a meeting with a nurse working in our service who will tell them what type of feeding and postoperative care their child will need and also what future procedures will be undertaken. To help them understand the situation better, they will then have an opportunity to observe another child who is being successfully treated for a similar problem.

\section{2 - 2 - Surgical treatment}

- The first step: repair of the soft
palate, the lip, and the nose (fig. 1 and 2)

We perform this first surgical procedure after the infant reaches the age of 3 months. We reconstruct the soft palate in accordance with the veloplasty technique that Kriens first described in 1967, which Sommerlad ${ }^{5}$ later renewed and improved. 


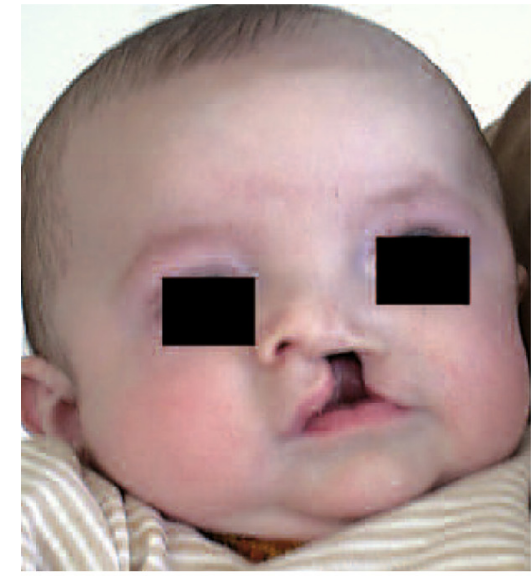

a

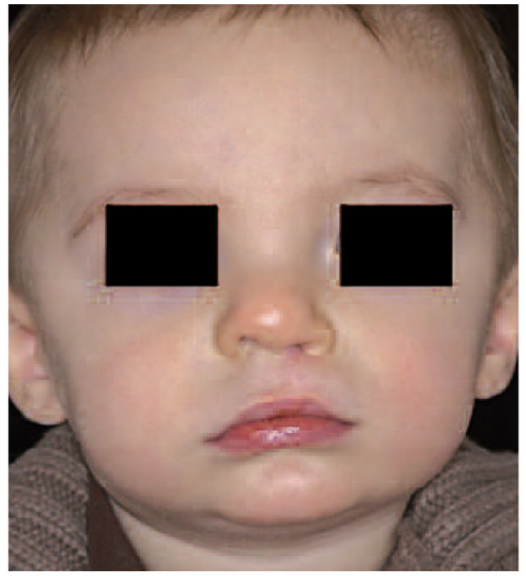

$\mathrm{b}$

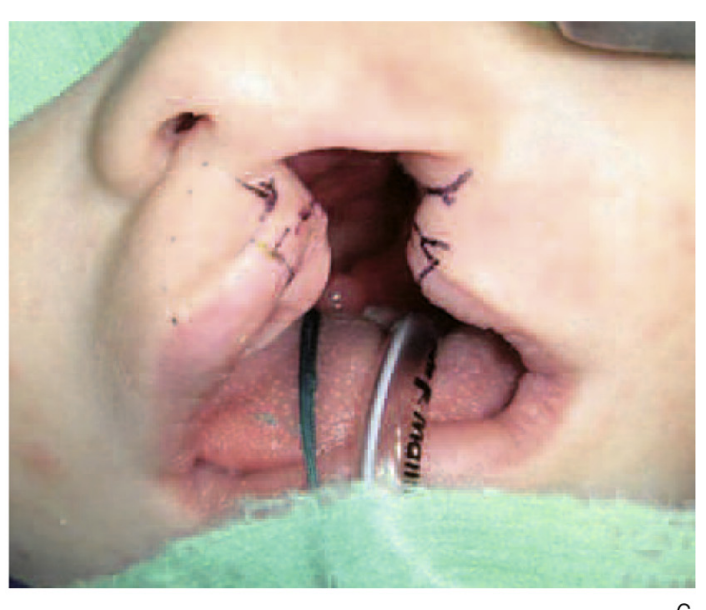

C

Figures 1 a to $c$

A left side unilateral cleft. The deformity before surgery (a) and 1 year later after combined cheiloplasty and rhinoseptoplasty (b). We plan the procedure in accordance with a modified Millard technique (c).
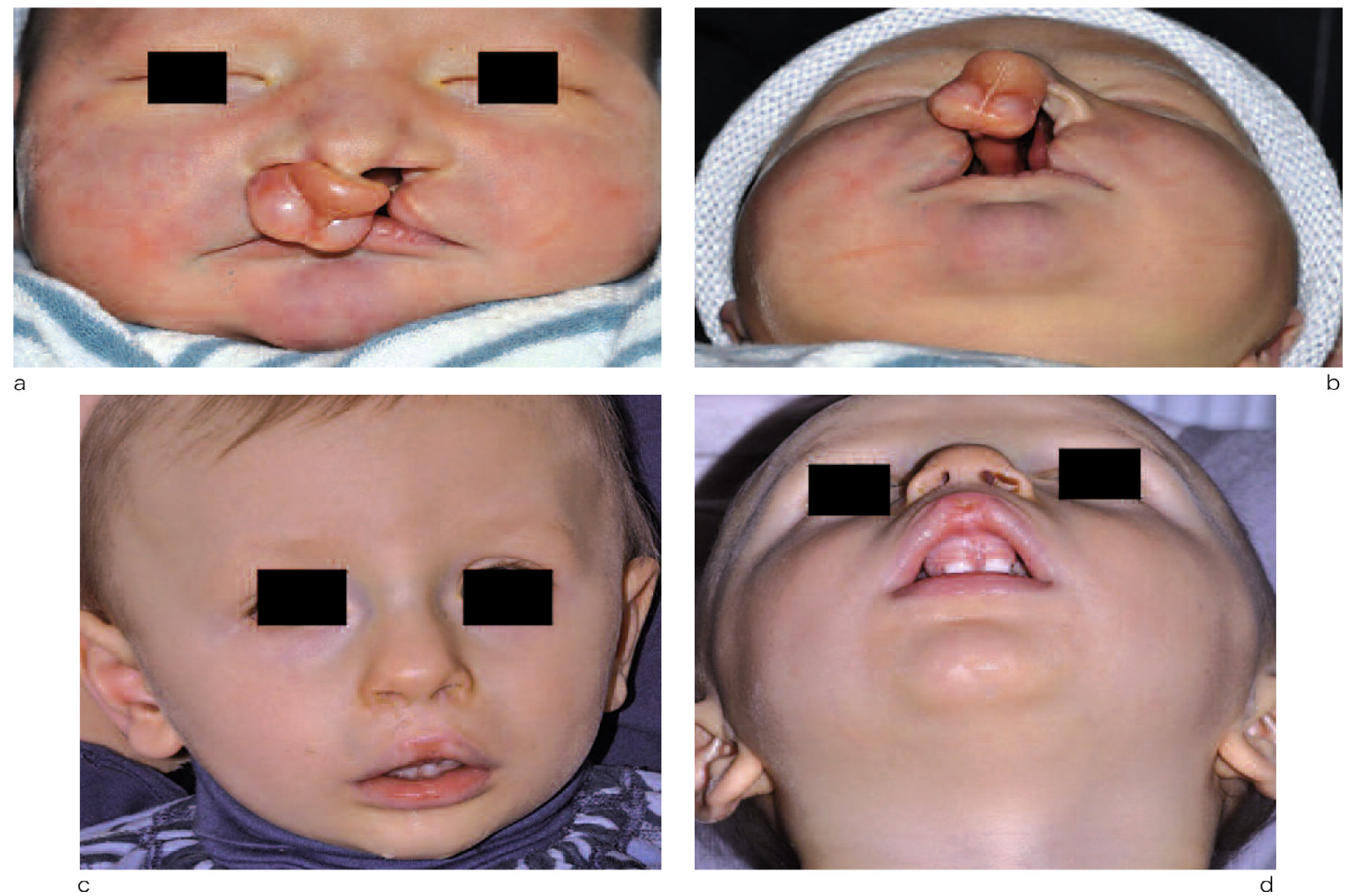

Figures 2 a to $d$

Bilateral labio-palatal cleft. Pre-operative views $(a, b)$ and post-operative views $(c, d)$. 
We incorporate an anatomically correct unilateral or bilateral cheiloplasty in this procedure using a modified Millard technique. At the same time we perform a rhino-septoplasty to reposition the alar cartilage and the nasal septum that are always deviated on the side opposite the cleft in its anterior sector. Here, muscle repair is essential because it will restore proper symmetry to the lips and give good posture to the tip of the nose. We affix nasal retainers, made on site by the Prosthetic Laboratory of our Service, in place with a trans-septal suture. We replace them with removable bi-nasal retainers, also prepared on site, that are worn for a total duration of four months.

After completion of the surgery, which lasts from 3 and a half hours to 5 hours, depending on the type of cleft, newborns are admitted to the Post-Intervention Observation Room where they are carefully watched, and given intravenous morphine analgesia and antibiotic and cortisone therapy. They are fed, beginning on the day of the operation by syringe or bottle spoon for three weeks to remove the possibility of trauma to the newly joined soft palate that bottle-feeding could cause. We use no splints or arm or other kinds of restraints on these children recovering from their first operation placing them instead in decubitus dorsal with head slightly raised so they have free movement and can suck a thumb or a pacifier.

\section{- Second surgical operation: clos- ing the osseous cleft (fig. 3)}

Patients are scheduled for this second operation when they are about 12 months old and the hard palate bone gap has had time to close appreciably spontaneously after the lip and soft palate repair.
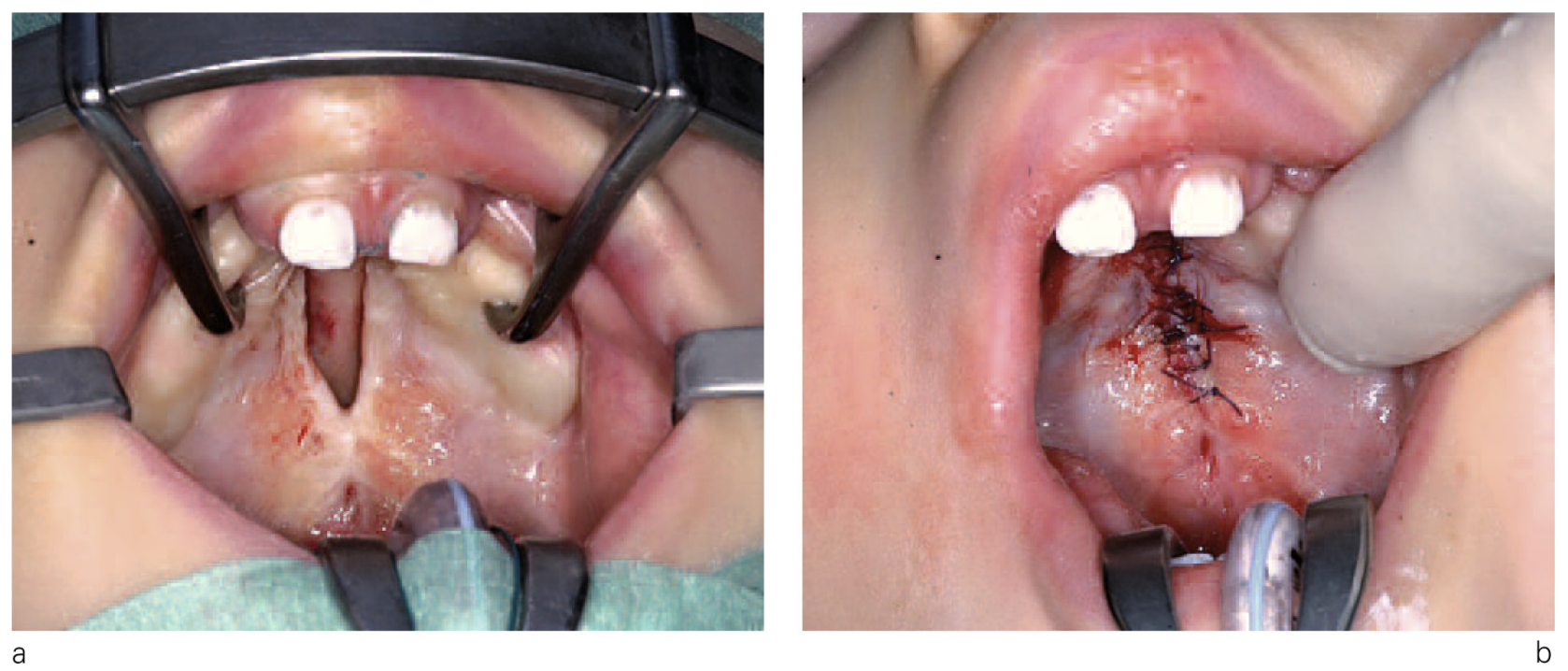

Figures $3 a$ and $b$

The appearance of the hard palate cleft at 12 months after the spontaneous shrinking of the gap that follows repair of the soft palate and lip at 3 months. Surgeons can now close the osseous defect without leaving any scar zone in the reunited palate. 
Thanks to this spontaneous improvement, surgeons can always close the remaining hard palate gap in two planes by sliding the nasal mucosa and the palatal fibromucosa together without leaving any denuded osseous zones. This is important because studies have clearly shown that if secondary scar tissue finds a place to form it can interfere with proper maxillary growth ${ }^{1}$. We close the osseous gap right up to the retroalveolar zone, leaving the alveolar cleft itself untouched, free of any surgical intervention at that time.

- Third surgical operation: gingivoperiosteoplasty combined with bone graft (fig. 4)

We close the alveolar cleft, which has been spared any scar tissue formation, when patients are 4 to 6 years old in the third and last primary cleft surgical procedure. It is undertaken after completion of orthodontic preparation that consisted of correction of the position of the retruded small alveolar fragment and, notably, repositioning of the canine through expansion provided either by a cemented or splint-born palatal expander or, earlier on younger patients, by a quad'helix cemented to temporary molars. Orthodontists decide which type of appliance is best suited to the special needs of the individual patient's malocclusion. After orthodontists have achieved an intercanine distance of about 32 to $35 \mathrm{~mm}$ for their patients they put a removable quad'helix in place that is worn until the surgical intervention begins.

Surgeons routinely place a graft of spongy iliac bone in position when they perform gingivoperiostoplasties. In our procedures, the mucosa, which is free of scar tissue, is of high quality making it possible for us assure a full, impermeable seal of both the oral and the nasal cavities. The graft must be placed vertically along the maxilla to the level of the piriform aperture but no higher. Surgeons should raise the mucoperiosteal flap generously so that it can cover the graft freely, without tension and accomplish the sub-periosteal dissection prudently to avoid harming any permanent tooth buds that may lie in nearby cortical bone.

When the surgery is complete the quad'helix is returned to its position and left in place to serve as a retainer for 3 to 6 months.

Orthodontists prepare the teeth of patients with bilateral clefts in the same way they treat patients with unilateral clefts. We do the surgery in two stages 2 to 6 months apart so as to avoid harming circulation in the mucosa of the premaxilla.

\section{2 - 3 - Associated and follow-up treatment for cleft patients}

For functional issues:

\section{- ENT}

It is important for ENT specialists to examine cleft palate patients at the age of nine months and then at least once a year systematically because they are at high risk for developing serious chronic otitis.

\section{- Speech-language pathology}

Speech therapists on our service, provide early "logopedic guidance" in 

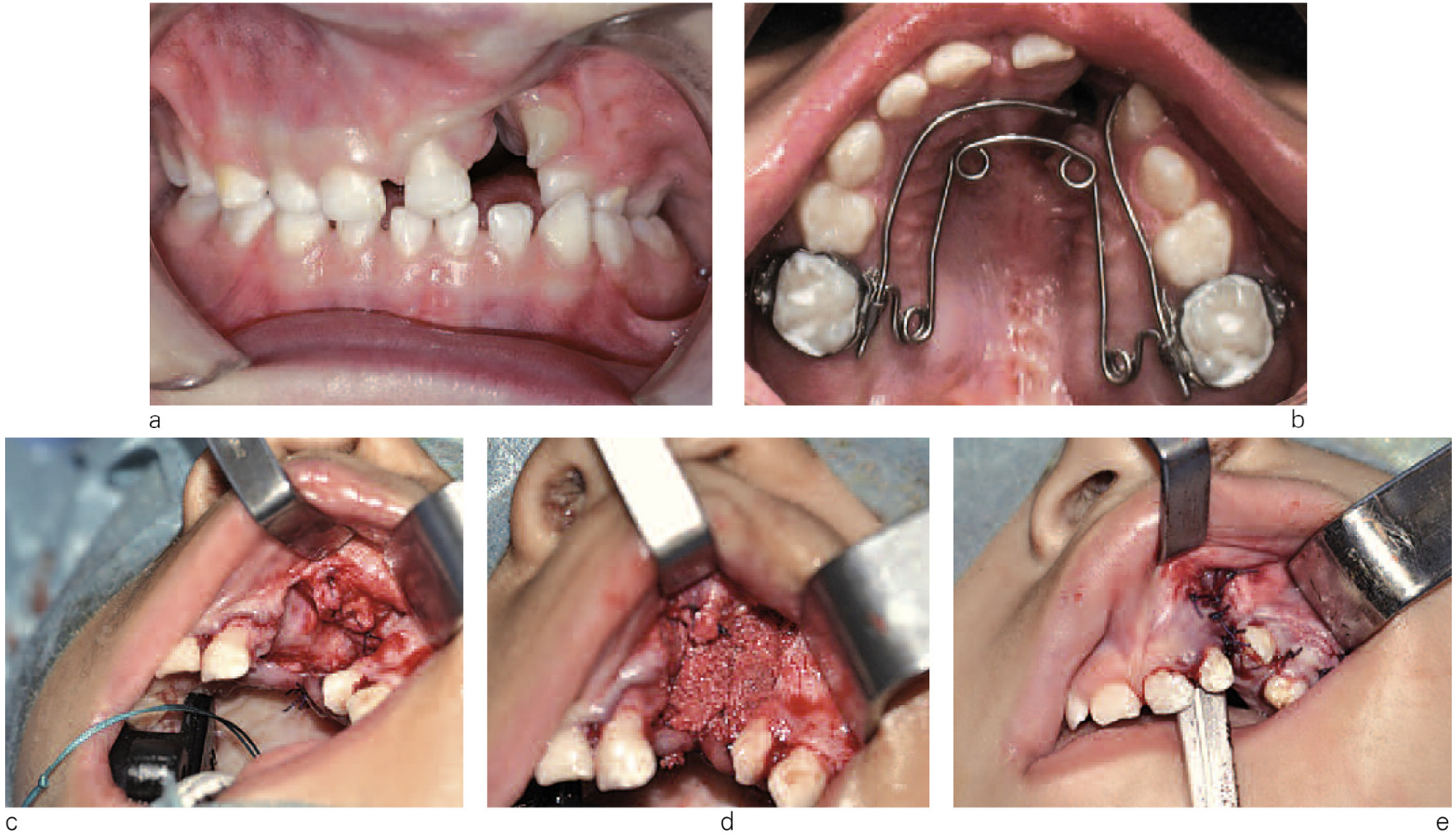

C
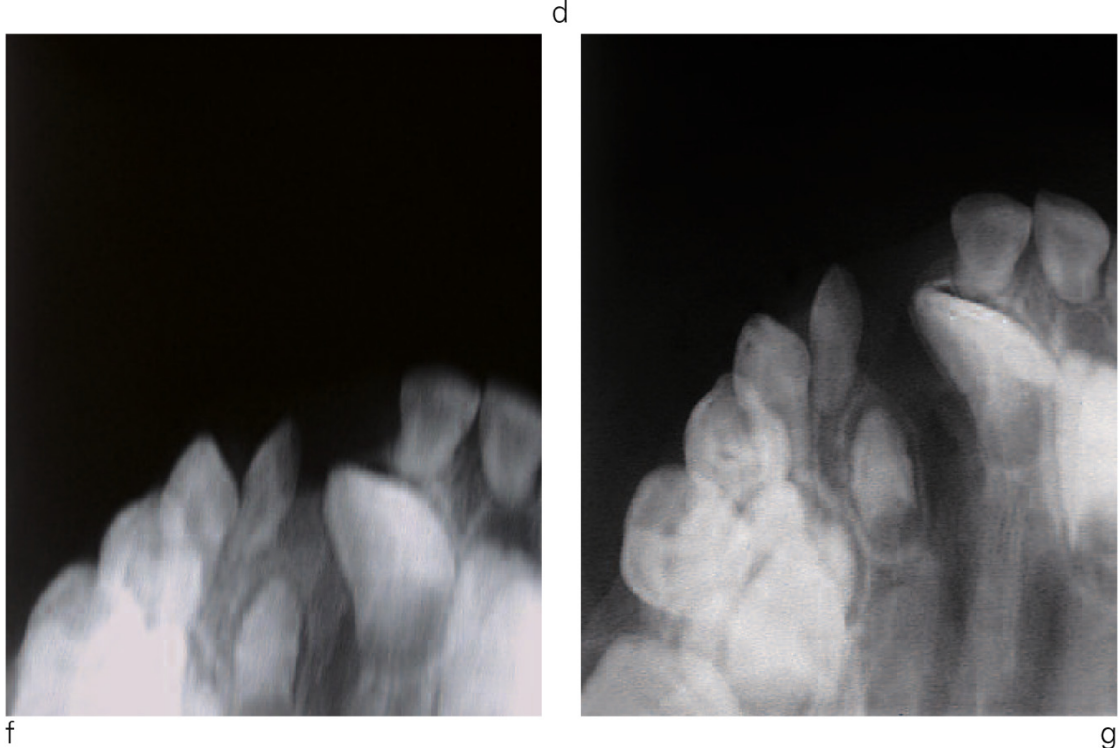

Figures 4 a to $\mathrm{g}$

$a$ and $b$ : intraoral frontal and palatal views of the cleft before surgery. A quadhelix has opened up an intercanine distance of 32 to $35 \mathrm{~mm}$.

$c$ to e: intraoral views of the gingivo-periosteal surgery combined with placement of a graft of spongy iliac bone. The closed nasal and palatal planes (c), the graft in place (d), and the sutured mucoperiosteal flap (e).

$f$ and g: occlusal $X$-ray films showing the lateral incisor bud emerging in the graft. 
three sessions to children of the same age with similar deformities and their parents. The meetings, which take place when patients are 12 to 16 months old, 18 to 26 months old, and 28 to 36 months old, are designed to acquaint parents with the importance of the "pre-phonation" phase, a time when it is possible to make a game of at home exercises that improve velo-pharyngeal competence. This approach also emphasizes for parents the critical role that careful auditory surveillance will play for their children.

When patients are 3 years old, speech pathologists make an auditory assessment, associated with a nasometric sensitivity and specificity evaluation that determine whether patients will simply continue with the breathing exercises they had been taught or will require more intensive speech therapy at home. Their progress is then evaluated annually.

\section{- Orthodontics}

Cleft palate patients often require interceptive orthodontic treatment to correct incisor malpositions as well as protrusions, retrusions, or cross bites. The permanent central incisor on the border of the cleft is often rotated sometimes by as much as $90^{\circ}$ - and inclined palatally enough to irritate and scar labial mucosa. It is at risk of being traumatized but severe rotations of unerupted teeth visible on panoramic $X$-rays sometimes correct themselves spontaneously during eruption. By correcting irregular teeth with a removable or a multi-band appliance, orthodontists can sometimes re-establish the anterior locking necessary for harmonious maxillo-mandibular growth. In their treatments, orthodon- tists must be careful to keep tooth roots fully surrounded by basal bone because any weakening of periodontal support can make the quality of the ultimate prognosis doubtful. The multidisciplinary treatment team must maintain especially vigilant supervision of patients with syndromic forms of clefts that are accompanied by multiple congenital absences of teeth. The collaborative team should discuss the problems of these patients who require case-by-case adaptions of treatment protocols.

\section{- Surgery}

Surgeons who are members of the multi-disciplinary team coordinate treatment of cleft palate patients from the prenatal period to the end of the growth period. Their first responsibility is to insure that the initial reparative operation produces optimal morphological and functional results with wellformed labio-nasal and velar muscle bands, fully installed nasal breathing, and maxillary arch continuity that will encourage permanent teeth to erupt in good position, and eventually establish a correct canine lock.

Depending on the wishes of the patient, the team will determine whether secondary operations are needed to deal with lip or cutaneous scarring. A rhinoplasty associated with a new septoplasty may be indicated to repair a nasal deformity insufficiently corrected by the initial surgery. The surgeon follows the same protocol that guided the first procedure seeking the objective of restoring the anatomy of the cartilages. Patients will again wear a conformer after the secondary rhino-septoplasty. Achieving good function for patients is crucial and requires ongoing collaborative 
communication between speech language therapists, orthodontists, and surgeons all working to prevent any interferences with correct growth and function such as encroachment of possible compensatory phenomena as well poor speech production that appointments with the speech language therapist can forestall. Surgeons will ascertain whether additional operations on the soft palate or pharynx are required to make sure students with treated clefts are not handicapped in their school work.

\section{2 - 4 - Surgical management of arrested skeletal growth}

We perform intermediate surgery of maxillary distraction with a technique most of whose components we developed at our Center using a hybrid, internal distractor with double bone and dental anchorage ${ }^{2,3}$. Indications for distraction therapy most often arise for patients with syndromic clefts not accompanied by a short, hypoplastic lip. Clinical syndrome forms of clefts, like the Van Der Woude syndrome, are classically accompanied by multiple agenesies of teeth and also frequently demonstrate over-all hypoplasia of the maxilla. Early osseous distraction of the upper jaw has the objective of establishing proper articulation with the mandible, which is usually normal size, so that the engagement of the two jaws will stimulate the maxilla to follow the normal growth pattern of its inferior fellow. Maxillary distraction also facilitates orthodontic alignment of the maxillary teeth with creation and maintenance of adequate space for unerupted teeth. And, finally, maxillary distraction improves facial appearance by correcting the position and bulk of the upper lip, all of which make it easier for cleft palate patients to integrate themselves in their lives at school and play. When there is a discrepancy greater than $10 \mathrm{~mm}$ between maxilla and mandible we believe intermediary maxillary distraction is indicated.

But this procedure does not mean that orthognathic surgery will not be needed at the termination of the growth period. What it does effectively is bolster cleft palate patients' self esteem at a critical juncture in their lives and limit the extent of the maxillary advancement required in the secondary, post growth surgery thus reducing the risk of relapse caused by the shrinking traction of soft tissue scarring. This progressive maxillary advancement also limits velo-pharyngeal decompensation tied to the advancement of the soft palate.

Orthodontists and oral surgeons jointly make the decision on what surgical correction will be required at the end of the growth period. On our service we stress the importance of maintaining the harmonious size and shape of the maxillary arch and of preserving space for unerupted upper lateral incisors so that implants can be placed where, and when, indicated. Orthodontists must also prepare the two arches so that a stable postoperative occlusion will be established, giving maximum protection against development of any future antero-posterior discrepancy. 


\section{$3-$ CONCLUSION}

We have derived the interventional calendar that we use on our service from the one Talmant described ${ }^{7}$. This so-called functional approach we believe is a perfectly logical and effective one because using it we can reduce the assault that scar formation levels on trouble-free growth of the maxilla. This calendar also has the objective of promoting the nasal breathing that we now fully understand plays an important role in the growth and balance of the middle third of the face.

\section{REFERENCES}

1. Benateau H, Diner PA, Soubeyr and E, Vazquez MP, Picard A. Les séquelles maxillaires dans les fentes labioalvéolopalatovélaires. Rev Stomatol Chir Maxillofac 2007;108:297300.

2. Picard A, Diner PA, Labbé D, Nicolas J, Tomat C, Seigneuric JB, Vazquez MP, Benateau $\mathrm{H}$. Les séquelles maxillaires dans les fentes labioalvéoloplatovélaires. Place de la distraction ostéogénique. Rev Stomatol Chir Maxillofac 2007;108:313-20.

3. Picard A, Diner PA, Galliani E, Tomat C, Vazquez MR, Carls FP. Five years experience with a new intraoral maxillary distraction device (RID). Br J Oral Maxillofac Surg. 2010 Dec 8.

4. Shaw WC, Semb G, Nelson P, Brattström V, Mølsted K, PrahlAndersen B, Gundlach KK. The Eurocleft project 19962000: overview. J Craniomaxillofac Surg 2001;29(3):131-40.

5. Sommerlad BC, Henley M, Birch M, Harland K, Moiemen N, Boorman JG. Cleft palate rerepair - a clinical and radiographic study of 32 consecutive cases. Br J Plast Surg 1994;47(6):406-10.

6. Talmant JC. Current trends in the treatment of bilateral cleft lip and palate. In: Precious Davis S, Cleft Lip and Palate: A physiological approach. Oral and Maxillofac Surg Clin North Am 2000;12:421-41.

7. Talmant JC, Talmant JC, Lumineau JP. Une approche fonctionnelle lors du traitement primaire des fentes labioalvéolopalatovélaires pour le minimum de séquelles. Rev Stomatol Chir Maxillofac 2007;108(4):255-63.

\section{Appointments}

- A. Picard, E. Galliani, V. Soupre, N. Kadlub, S. Cassier, G. Constantinescu, F. Zazurca, C. Tomat, Vi-Fane, C. Trichet-Zbinden, C. Chapuis-Vandenbogaerde, P.A. Diner, M.-P. Vazquez

AP-HP, Armand-Trousseau Children's Hospital, Maxillo-facial Surgery and Plastic surgery Service; Referral Center for Rare Deformities off the Face and the Oral Cavity

- A. Picard, N. Kadlub, S. Cassier, M.P. Vazquez; Pierre and Marie Curie-Paris 6, UFR de Medicine Pierre and Marie Curie, Paris, F-75005. 